\title{
Prader-Willi syndrome identified by methylation specific multiplex ligation dependent probe amplification (MS-MLPA)
}

\author{
Simona Loredana Vasilache ${ }^{1,2}$, Adelina Micheu², Claudia Banescu ${ }^{3}$, Valeriu Moldovan ${ }^{3}$, \\ Carmen Duicu', Ionela Maria Pascanu ${ }^{2,4}$, Oana Marginean ${ }^{1}$ \\ ${ }^{1}$ Department of Pediatrics, University of Medicine and Pharmacy, Tg. Mures, Romania \\ ${ }^{2}$ Department of Endocrinology, Mures County Hospital, Tg. Mures, Romania \\ ${ }^{3}$ Department of Genetics, University of Medicine and Pharmacy, Tg. Mures, Romania \\ ${ }^{4}$ Department of Endocrinology, University of Medicine and Pharmacy, Tg. Mures, Romania
}

\begin{abstract}
Prader-Willi syndrome (PWS) is a multisystemic complex genetic disorder caused by lack of expression of genes on the paternally inherited chromosome 15q11.2-q13 region. There are three major genetic subtypes in PWS: paternal 15q11-q13 deletion (70\% of cases), maternal uniparental disomy $15(25-30 \%)$ and imprinting defect (1-3\%). The clinicians confront the challenge of discern more clearly between the classic PWS and the various PWS-like syndrome (PWLS). It is necessary to study these issues at the molecular level to explain these genetic similarities and to provide appropriate genetic counseling and treatment. We present a case of a 6 years old male patient with severe hypotonia, feeding difficulties in neonatal period, developmental delay in neuromotor acquisition, hyperphagia and obesity (BMI: +4.66 SD). The genetic analysis methylation specific multiplex ligation dependent probe amplification (MS-MLPA) revealed an aberrant methylation of $\mathrm{CpG}$ island. It is important to mention that a precise diagnosis of PWS and an early multidisciplinary approach are essential for efficient long-term management, for preventing complications and improve quality of life in this patients.
\end{abstract}

Keywords: Prader-Willi syndrome, imprinting center, obesity

\begin{abstract}
Abbreviations
PWS - Prader-Willi syndrome

PWLS - PWS-like syndrome

BMI - body mass index

DS - standard deviation
\end{abstract}

MS-MLPA - methylation specific multiplex

ligation dependent probe amplification

SNRPN - small nuclear ribonucleoprotein-associated

protein $\mathrm{N}$

\section{INTRODUCTION}

Prader-Willi syndrome (PWS) is a disorder characterized by neurogenetic, neurometabolic and neurobehavioral alterations. It has an estimated prevalence worldwide in the range of 1 in 10,000 to 30,000 individuals (1). PWS is a genetic condition due to paternal loss of imprinted genes on chromosome 15 and characterized by a range of mental and physical findings including obesity that can be lifethreatening (2). Approximately $70 \%$ of individuals
IGF1 - insulin growth factor 1

TSH - thyroid stimulating hormone

FT4 - free thyroxine

NDN gene - the encoding gene of necdin

DNA - deoxyribonucleic acid

IC - imprinting center

with PWS are caused by a non-inherited deletion in the paternally derived chromosome 15q11-q13 region; about $25 \%$ result from maternal disomy 15 and in less than $3 \%$ of cases are individuals genomic imprinting defects (2-4).

Affected infants constantly have significant hypotonia, feeding difficulties, and failure to thrive, followed in early childhood by excessive appetite with gradual development of obesity, short stature, intellectual disabilities and behavioral problems (5). 
In PWS a precocious multidisciplinary approach is fundamental to prevent complications, to improve quality of life and prolong life expectancy.

\section{CASE PRESENTATION}

In our department we had the opportunity to observe the case of a child from a non-consanguine- ous couple, a 6 years old male patient, from an abnormal pregnancy-oligoamnios. $\mathrm{He}$ presented diminished fetal activity and he was born at 42 weeks by cesarean section, with a birth weight of $2,100 \mathrm{~g}(-2.18 \mathrm{SD})$, a birth height of $47 \mathrm{~cm}(-1.63$ SD) and APGAR score 6/1 minute. During the neonatal period he presented severe hypotonia, feeding difficulties (until 3 weeks of age the child was fed

\begin{tabular}{|c|c|c|c|c|c|c|c|}
\hline$D$ [nt] & Gene-Exon & Chr.band & hg18 loc. & Height & Area & Ratio $^{H}$ & Stdev \\
\hline 154 & TUBGCP5-8 & $5 \mathrm{q} 11.2$ & $15-020.398303$ & 8222 & 49296 & 0.92 & 0.03 \\
\hline 434 & NIPA1-4 & & $15-020.612289$ & 396 & & 0.05 & \\
\hline 172 & MKRN3-1 & & $15-021.362818$ & 8488 & 54621 & 0.96 & 0.03 \\
\hline 409 & MAGEL2-1 & g11.2 & $15-021.440355$ & 7605 & 57910 & 1 & 0.03 \\
\hline 445 & & & $5-021.482381$ & 7083 & & 0.99 & 0.03 \\
\hline 419 & NDN-1 [HHA1] & $15 q 11.2$ & $15-021.483412$ & 648 & 5436 & 0.08 & 0 \\
\hline 287 & SNRPN-u1b & & $15-022.619902$ & 8058 & 51562 & 1.01 & 0.03 \\
\hline 238 & SNRPN-u1b* & & $15-022.626072$ & 12545 & & 0.99 & 0.03 \\
\hline 278 & SNRPN-Intr.u2 & $15 q 11.2$ & $15-022.690980$ & 8852 & 57458 & 0.99 & 0.03 \\
\hline 270 & SNRPN-Intr.u2 & & $15-022.703328$ & 10268 & 64664 & 0.98 & 0.03 \\
\hline 256 & SNRPN-U5 & & $15-022.716714$ & 10000 & 61727 & 0.92 & 0.03 \\
\hline 391 & SNRPN-U5 & & $15-022.717321$ & 8033 & & 0.94 & 0.03 \\
\hline 250 & SNRPN-CpG is & & $15-022.751105$ & 819 & 4598 & 0.06 & 0 \\
\hline 178 & SNRPN-CpG isI & & $15-022.751214$ & 0 & 0 & 0 & 0 \\
\hline 190 & SNRPN-CpG isI & & 1480 & 1057 & 6430 & 0.13 & 0 \\
\hline 142 & SNRPN-CpG is $/$ & & $15-022.751773$ & 1143 & & 0.15 & 0 \\
\hline 294 & $\mathrm{APN}_{3} 3$ & & 4248 & 8954 & & 1.04 & 0.03 \\
\hline 400 & SNRPN-7 & & $15-022.772555$ & 8958 & 65303 & 0.93 & 0.03 \\
\hline 214 & SNRPN-HB2-85- & & $15-022.848250$ & 17955 & 109877 & 1.04 & 0.03 \\
\hline 472 & SNRPN-HB2-85- & & 22.872658 & 8189 & 68867 & 0.94 & 0.03 \\
\hline 328 & SNRPN-HB2-85- & & $15-022.888662$ & 1321 & 8642 & 0.11 & 0 \\
\hline 355 & A-13 & & & 8545 & & 0.94 & 0.03 \\
\hline 301 & $A-8$ & & & 10698 & & 1.02 & 0.03 \\
\hline 160 & BA-7 & & 740 & 10364 & & 0.9 & 0.03 \\
\hline 197 & A-6 & & & 11132 & & 1.03 & 0.03 \\
\hline 73 & $3 A-5$ & & 674 & 8878 & 62548 & 1 & 0.03 \\
\hline 184 & E3A-1 [HHA1] & & 184 & 0 & 0 & & 0 \\
\hline 366 & TP10A-5 & & 522207 & 10569 & 71690 & 1.01 & 0.03 \\
\hline 226 & $0 \mathrm{~A}-1$ & & & 9988 & 169 & 1 & 0.03 \\
\hline 220 & B3-12 & & 1242 & 8575 & 56340 & 1.03 & 0.03 \\
\hline 382 & B3-10 & & & 429 & & 0.04 & 0 \\
\hline 202 & A2-14 [HHA & & 749 & 404 & 24 & 0.04 & 0 \\
\hline 463 & MLH1-1 [HHA1] & & 9621 & 0 & 0 & 0 & 0 \\
\hline 346 & BLM-1 [HHA1] & & & 0 & 0 & 0 & 0 \\
\hline 427 & ence $\mathrm{C} / \mathrm{M}^{*}$ & & & 8213 & & 1.01 & 0.03 \\
\hline 244 & ence $\mathrm{C} / \mathrm{M}^{*}$ & & 9132 & 12259 & 74491 & 0.96 & 0.03 \\
\hline 264 & & & & & & 1.01 & 0.03 \\
\hline 136 & Reference $\mathrm{C} / \mathrm{M}^{*}$ & & & 10862 & 69602 & 1.01 & 0.03 \\
\hline 454 & ence $\mathrm{C} / \mathrm{M}^{*}$ & & & & & 1.03 & 0.03 \\
\hline 130 & $M^{*}$ & & & & & 0.95 & 0.03 \\
\hline & eference $\mathrm{C} / \mathrm{M}^{*}$ & & & 9730 & & 0.99 & 0.03 \\
\hline 166 & eference $\mathrm{C} / \mathrm{M}^{*}$ & & & 13590 & & 1 & 0.03 \\
\hline 208 & ence $\mathrm{C} / \mathrm{M}^{*}$ & & 475 & 12938 & 78148 & 0.93 & 0.03 \\
\hline 337 & ference $\mathrm{C} / \mathrm{M}^{*}$ & & & & & 0.95 & 0.03 \\
\hline 148 & ference $\mathrm{C} / \mathrm{M}^{*}$ & & & 12462 & 73258 & 0.92 & 0.03 \\
\hline 232 & ence $\mathrm{C} / \mathrm{M}^{*}$ & & & 11523 & & 1.02 & 0.03 \\
\hline 480 & Reference C/M* & & & 8434 & & 1.05 & 0.03 \\
\hline 309 & Reference $\mathrm{C} / \mathrm{M}^{*}$ & $22 q 12.2$ & $22-02$ & 11243 & 72672 & 1 & 0.03 \\
\hline & 865 & 61220 & 0.99 & 0.03 \\
\hline
\end{tabular}

FIGURE 1. Sample report of the data obtained by capillary electrophoresis from a DNA sample analysed with SALSA MS-MLPA probemix ME028 Prader Willi/Angelman probemix. With red are indicated the SNRP-CpG island which are hypomethylated 
by nasogastric tube)and developmental delay in neuromotor acquisition. Later the patient presented hyperphagia with important weight gain and behavioral problems - temper tantrumps and stubbornness. Karyotype from fresh blood was 46,XY. Afterwards a molecular genetic analysis was performed (methylation specific multiplex ligation dependent probe amplification MS-MLPA by using SALSA MS-MLPA ME028 kit) revealing an aberrant methylation of $\mathrm{CpG}$ island, which belongs to SNRPN promoter (Fig. 1).

Thereafter, he is admitted to our clinic; physical examination reveals dysmorphic features (narrowed forehead, almond shaped eyes, thinner upper lip), delayed motor and cognitive development, decreased stature (height: -1.05 SD), excessive weight gain (BMI: +4.66 SD) and under development of the genitalia.

Associated comorbidities: congenital microcephaly, cytomegalovirus infection of the central nervous system, severe psychomotor and cognitive retardation, surgically corrected cryptorchidism.

The laboratory investigation revealed IGF1: $62.89 \mathrm{ng} / \mathrm{ml}$, TSH: $1.376 \mu \mathrm{UI} / \mathrm{ml}$, FT4: $0.94 \mathrm{ng} / \mathrm{dl}$ and elevated liver enzymes. The abdominal ultrasound revealed: unilateral hydronephrosis, hepatic steatosis and hepatomegaly. The severe obstructive sleep apnea was diagnosed; the polysomnography revealing apneea-hypopnea score $(\mathrm{AHI})>40$. The adenoidectomy for adenoid vegetation was performed and the post-procedure AHI was 9.4. After adenoidectomy growth hormone therapy ( $\mathrm{rhGH}$ ) was initiated. rhGH doses were progressively increased, and after 6 months of treatment an improvement is noted in height: $-0.46 \mathrm{SD}, \mathrm{BMI}:+3.82$ $\mathrm{SD}$ and height velocity: $9 \mathrm{~cm} /$ year.

\section{DISCUSSIONS}

It is known that PWS has common clinical aspects with other diseases, which makes it difficult to precisely diagnose. Therefore, the clinicians confront the challenge of distinguish more clearly between the classic PWS and the various PWS-like syndrome (PWLS). It is necessary to study these issues at the molecular level to explain these genetic similarities and to provide appropriate genetic counseling and treatment. PWLS share elements of the PWS phenotype and the gene functions disrupted in PWLS are likely to be involved in genetic pathways that are significant for the expresion of PWS phenotype (6).

Considering these aspects, it is important to choose the correct genetic test to identify patients with classic PWS because Rocha et al. (7) reveals in a meta-analysis other mutation associated with PWS-like phenotype, such us: deletion of 6q, paracentric inversion, fragile $\mathrm{X}$ syndrome.

DNA methylation is considered a solid tool to determine paternal-only, maternal-only, or biparental (normal) inheritance, but can not differentiate between maternal uniparental disomy 15 or imprinting defects (5). Compared to conventional DNA methylation, a new semi-quantitative method, namely "methylation-specific multiplex-ligation probe amplification" (MS-MLPA) is recommended for methylation detection in imprinting disorders (ex. Beckwith Wiedemann syndrome, Silver Russel syndrome) and uniparental disomy. MS-MLPA will determine the methylation status by using 5 methylation specific probes (4 for $S N R P N$ gene and one for NDN gene) but also detect the copy number changes (deletions on chromosome 15q11), therefore being indicated to establish the diagnosis of PWS (8.9).

For this reason, MS-MLPA analysis might be considered as the first testing when suspecting PWS as a possible diagnosis (10).

Following the MS-MLPA test, our patient was diagnosed with PWS due to the aberrant methylation of the $\mathrm{CpG}$ islands belonging to the SNRPN promoter, and thus $15 \mathrm{q}$ deletions were ruled out. Unfortunately, because the technique can not distinguish between imprinting defects and chromosome 15 maternal uniparental disomy, additional tests, like DNA polymorphism analysis may be performed in order to indicate the exact cause of the disease, but nevertheless the PWS diagnosis is already established.

Understanding the particular genetic etiology in patients with PWS is essential for the correct genetic counseling of affected families. Genetic defect which produces PWS is correlated with evaluation of recurrent risk (11).

Imprinting defects are a rare but significant cause of PWS. Patients with an imprinting defect have apparently normal chromosome 15 of parental and maternal origin, but present aberrant DNA methylation of SNRPN promoter. When counseling patients with a detected imprinting defect, a recurrence risk is up to $50 \%$, because the mutation is likely dominant and occurred in the paternal grandmother's germ line (12); those without an IC deletion would be expected to have a lower risk.

Maternal uniparental disomy of chromosome 15 is the second cause of PWS and present also aberant methylation frequently de novo. This etiology has a recurrence $<1 \%$ (2). 


\section{CONCLUSIONS}

It is important to mention that a precise diagnosis of PWS and an early multidisciplinary approach are essential for efficient long-term management, to prevent complications and improve the quality

\section{REFERENCES}

1. Whittington J., Holland A., Webb T. et al. Cognitive abilities and genotype in a population-based sample of people with Prader-Willi syndrome. J. Intellect. Disabil. Res. 2004; 48:172-187.

2. Cassidy S.B., Driscoll D.J. Prader-Willi syndrome. Eur. J. Hum. Genet. 2009; 17(1):3-13.

3. Ohta T., Gray T.A., Rogan P.K. et al. Imprinting-mutation mechanisms in Prader-Willi syndrome. Am. J. Hum. Genet. 1999; 64(2):397-413.

4. Bittel D.C., Butler M.G. Prader-Willi syndrome: clinical genetics, cytogenetics and molecular biology. Expert. Rev. Mol. Med. 2005; 7(14): 1-20.

5. Butler M.G., Lee P.D.K., Whitman B.Y. Management of Prader-Willi syndrome. 3rd. ed. New York: Springer. 2006.

6. Cheon C.K. Genetics of Prader-Willi syndrome and Prader-Will-like syndrome. Ann Pediatr Endocrinol Metab. 2016; 21(3):126-135

7. Rocha C.F, Paiva C.L.A. Prader-Willi-like phenotypes: a systematic review of their chromosomal abnormalities. Genetics and Molecular Research. 2014; 13:2290-2298. of life.The genetic basis of this rare disorder differ but MS-MLPA is a simple, rapid and useful method for identification of PWS cases with aberrant methylation status.

Conflict of interest: none declared Financial support: none declared

8. Henkhaus R.S., Kim S.J., Kimonis V.E. et al. Methylation-specific multiplex ligation-dependent probe amplification and identification of deletion genetic subtypes in Prader-Willi syndrome. Genet. Test. Mol. Biomarkers. 2012; 16(3):178-186.

9. Angulo M.A., Butler M.G., Cataletto M.E. Prader-Willi syndrome: a review of clinical, genetic, and endocrine findings. J Endocrinol Invest. 2015; 38:1249-1263.

10. Pagliardini S., Ren J., Wevrick R. et al. Developmental abnormalities of neuronal structure and function in prenatal mice lacking the prader-willi syndrome gene necdin. Am J Pathol 2005; 167:175-91

11. Cassidy S.B., Schwartz S., Miller J.L. et al. Prader-Willi syndrome. Genet Med 2012; 14:10-26.

12. Camprubí C., Coll M.D., Gabau E. et al. Prader-Willi and Angelman syndromes: genetic counseling. Eur J Hum Genet 2010; 18:154-5. 